\title{
Video Article \\ Antimicrobial Characterization of Advanced Materials for Bioengineering Applications
}

\author{
Miguel Marti* ${ }^{1}$, Belén Frígols ${ }^{* 1}$, Angel Serrano-Aroca ${ }^{1}$ \\ ${ }^{1}$ Facultad de Veterinaria y Ciencias Experimentales, Universidad Católica de Valencia San Vicente Mártir \\ * These authors contributed equally
}

Correspondence to: Angel Serrano-Aroca at angel.serrano@ucv.es

URL: https://www.jove.com/video/57710

DOI: doi: $10.3791 / 57710$

Keywords: Bioengineering, Issue 138, Advanced materials, antimicrobial, characterization, disk diffusion, surface material, bioengineering

Date Published: 8/4/2018

Citation: Martí, M., Frígols, B., Serrano-Aroca, A. Antimicrobial Characterization of Advanced Materials for Bioengineering Applications. J. Vis. Exp. (138), e57710, doi:10.3791/57710 (2018).

\section{Abstract}

The development of new advanced materials with enhanced properties is becoming more and more important in a wide range of bioengineering applications. Thus, many novel biomaterials are being designed to mimic specific environments required for biomedical applications such as tissue engineering and controlled drug delivery. The development of materials with improved properties for the immobilization of cells or enzymes is also a current research topic in bioprocess engineering. However, one of the most desirable properties of a material in these applications is the antimicrobial capacity to avoid any undesirable infections. For this, we present easy-to-follow protocols for the antimicrobial characterization of materials based on (i) the agar disk diffusion test (diffusion method) and (ii) the ISO 22196:2007 norm to measure the antimicrobial activity on material surfaces (contact method). This protocol must be performed using Gram-positive and Gram-negative bacteria and yeast to cover a broad range of microorganisms. As an example, 4 materials with different chemical natures are tested following this protoco against Staphylococcus aureus, Escherichia coli, and Candida albicans. The results of these tests exhibit non-antimicrobial activity for the first material and increasing antibacterial activity against Gram-positive and Gram-negative bacteria for the other 3 materials. However, none of the 4 materials are able to inhibit the growth of Candida albicans.

\section{Video Link}

The video component of this article can be found at https://www.jove.com/video/57710/

\section{Introduction}

Implant failure is often a consequence of microbial infections that occur in spite of antimicrobial prophylaxis and aseptic working conditions. This problem is causing very high healthcare costs and is distressing among patients ${ }^{1}$. Important bacteria such as Staphylococcus aureus are currently considered to be very dangerous pathogens in nosocomial infections associated with catheters and other medical implants and are the main contaminants of medical instruments ${ }^{2}$. Therefore, the development of novel antimicrobial strategies is urgently needed for both daily and medical uses.

Antimicrobial agents include antibiotics ${ }^{3}$, quaternary ammonium compounds ${ }^{4}$, metal ions/oxides ${ }^{5}$, and antimicrobial peptides $\left(\right.$ AMPs) ${ }^{6}$. Antibiotics are gradually becoming less efficient due to bacterial resistance ${ }^{7}$, which is on the rise due to antibiotic overuse ${ }^{8}$. Quaternary ammonium compounds are only very efficient for a short-term use because of microbial resistance ${ }^{9}$. Metal ions/oxides have long been utilized as very effective antimicrobial agents and are used in many common commercial products including bandages, water filters, paints, etc. ${ }^{10,11,12}$. However, it has been demonstrated that these types of compounds can be toxic to some types of mammalian cells ${ }^{13}$.

AMPs show excellent antimicrobial and immunomodulatory properties ${ }^{14,15}$, and bacteria seem to find it very difficult to develop a resistance against them ${ }^{16}$. However, the process to produce pure AMPs is expensive; therefore, a large-scale production is not viable. Thus, strategies to counter the problems in producing AMPs have been developed (e.g., small molecular antibacterial peptoid mimics ${ }^{17}$, peptoids ${ }^{18}, \alpha-$ peptides $^{19}$ and $\beta$-peptides $\left.{ }^{20}\right)$. Methacrylate-ended polypeptides and polypeptoids have been synthesized for antimicrobial and antifouling coatings ${ }^{21}$.

The development of new antimicrobial agents such as advanced materials in pure or hybrid form, able to prevent and treat multidrug-resistant infections, is increasingly necessary. A broad range of new advanced materials for many bioengineering fields such as tissue and bioprocess engineering have been developed with improved chemical and physical properties in the last decades through several methods: plasmapolymerization grafting onto a hydrophobic substrate ${ }^{22,23,24}$, tailoring of crosslinking density ${ }^{25,26}$, polymerization in solution ${ }^{27,28,29,30}$, porogen dissolution ${ }^{31,32}$, and by the incorporation of nanomaterials such as graphene oxide $(\mathrm{GO})^{33,34,35,36}$ and carbon nanofibers (CNFs) ${ }^{37}$.

The study of the antimicrobial capacity of these new materials could exponentially increase their potential bioengineering applicability and has, therefore, become essential. We present an easy-to-follow protocol to quantify the antimicrobial activity of such new advanced materials. Here, 
after the sample preparation, two complementary methods are followed: the first is based on the agar disk diffusion test ${ }^{38}$ (diffusion method) and the second is based on the ISO $22196: 2007$ norm $^{39}$ to measure the antimicrobial activity on material surfaces (contact method).

\section{Sample Preparation}

1. Cut the material samples into $10 \mathrm{~mm}$ diameter disks with a $10 \mathrm{~mm}$ diameter cylindrical punch. NOTE: Brittle materials can be softened in suitable sterile solvents for $1 \mathrm{~h}$ and then cut into disks. For example, hydrophilic materials such as zinc alginate were tested following this protocol and were moistened in autoclaved water before cutting them in order to avoid sample breaking. However, other hydrophobic materials such as poly(3-hydroxybutyrate-co-3-hydroxyvalerate) do not need any kind of previous swelling in order to be properly cut.

2. Dry the sample material disks at $60^{\circ} \mathrm{C}$ in a vacuum oven $\left(<10^{-2}\right.$ Torr $)$ for $24 \mathrm{~h}$.

NOTE: Some materials may need a high drying temperature. However, it is important not to reach a temperature that could thermally degrade the material.

3. Measure the material film thicknesses with a digital caliper.

NOTE: This protocol recommends the utilization of material films with constant and similar thicknesses when comparing the antimicrobial activity of different materials.

4. Sterilize each specimen by immersion in ethanol $70 \%$ for $10 \mathrm{~min}$ and subsequent ultraviolet (UV) radiation for $1 \mathrm{~h}$ per each side. NOTE: UV radiation can be performed placing each specimen in a sterile Petri dish inside a laminar flow hood with a $12.0 \mathrm{~W}$ lamp of UV-C radiation.

CAUTION: Researchers should not expose themselves to UV radiation, because it is mutagenic.

5. Perform steps 1.1,1.2, 1.3 and 1.4. with the control material disks.

NOTE: Polyethylene terephthalate (PET) or alternative non-antimicrobial materials could be used as control disks in the diffusion and contact method. Furthermore, when characterizing nanocomposites or treated materials, the base material should be used as control material.

\section{Recommended Microorganisms}

NOTE: We recommend the use of 3 different microorganisms to study the antimicrobial capacity of the tested material against a wide range of microorganisms.

1. Use pure cultures of 3 microorganisms: the Gram-positive bacteria Staphylococcus aureus, the Gram-negative bacteria Escherichia coli, and the yeast Candida albicans.

NOTE: Other microorganism species can also be tested with this protocol by modifying the incubation conditions if necessary.

CAUTION: The required biosafety measurements must be followed according to the type of microorganism employed in this protocol.

2. Work with pre-sterile or autoclaved material and use a Bunsen burner during the whole process of the microbial manipulation or a biological safety cabinet (if necessary) to ensure aseptic conditions.

NOTE: Recommended autoclave conditions are $121^{\circ} \mathrm{C}$ during 15 min for culture mediums and $121^{\circ} \mathrm{C}$ during 20 min for the working material and biological residues.

\section{Agar Disk Diffusion Test (Diffusion Method)}

NOTE: When a liquid diffusion of antimicrobial compounds might be the main antimicrobial mechanism of advanced materials, the diffusion method can provide very useful information about the antimicrobial capacity of these materials. The material disk located at the center of the agar plate can form a transparent ring zone (halo) where a growth inhibition of microorganisms occurs after $24 \mathrm{~h}$ of culture (see Figure 1).

\section{Diffusion test procedure}

1. Prepare and autoclave tryptic soy agar (TSA) following the manufacturer's instructions.

2. Pour the TSA into sterile Petri dishes under aseptic conditions using a Bunsen burner or a laminar flow hood. NOTE: The TSA plates must be $4-6 \mathrm{~mm}$ thick.

3. Culture the different microorganisms to be tested aerobically for $18-24 \mathrm{~h}$ in the Petri dishes with TSA in an incubator at $37^{\circ} \mathrm{C}$.

4. Prepare and autoclave tryptic soy broth (TSB) following the manufacturer's instructions.

5. Pour the TSB in a $50 \mathrm{~mL}$ pre-sterilized centrifuge tube with a pre-sterilized serological pipette under aseptic conditions using a Bunsen burner or a laminar flow hood.

6. Resuspend a few colonies from step 3.1 .3 in $25 \mathrm{~mL}$ of TSB contained in a sterile centrifuge tube using a sterile cotton swab and vortex them for 1 min to achieve a uniform mixing.

7. Adjust the absorbance (at $540 \mathrm{~nm}$ ) of the culture with a spectrophotometer to the suitable number of colony forming units (CFU) per $\mathrm{mL}$ : approximately $1.5 \times 10^{8} \mathrm{CFU} / \mathrm{mL}$ for bacteria and from $1 \times 10^{6}$ to $5 \times 10^{6} \mathrm{CFU} / \mathrm{mL}$ for yeast.

NOTE: The culture and cuvette volumes to measure the absorbance must be selected according to the type of spectrophotometer utilized.

8. Vortex the microbial broth for 5 seconds to improve the microorganism dispersion and briefly submerge a sterile cotton swab in this microbial suspension. Remove the excess of liquid from the swab by pressing it against the tube wall containing the culture.

9. Evenly streak the microbial broth suspension with the sterile cotton swab onto the surface of the TSA plates in 3 planes to cover their whole surface with the microorganism and let it dry for 5 min after the inoculation.

NOTE: To remove any moisture, the TSA plates must be placed open in an inverted position at $37^{\circ} \mathrm{C}$ for $10-15$ min before the inoculation. 
10. Sterilize a pair of tweezers by immersing them in a beaker with ethanol $96 \%$ and then flaming them with a Bunsen burner or alcohol burner.

11. Place the sample disks to be tested and control disk at the center of the TSA plates using the pair of sterile tweezers.

12. Incubate aerobically the TSA plates in an inverted position at $37^{\circ} \mathrm{C}$ for $24 \mathrm{~h}$.

NOTE: In order to avoid any contamination risk, it is recommended to incubate the TSA plates in an inverted position. However, if the sample disk detaches from the TSA plates, do not perform this step in an inverted position. In this case, it is recommended to dry the plates in the laminar flow hood. This antimicrobial test must be performed at least in quadriplicate on different days to ensure reproducibility.

\section{Checking of microbial suspension concentration and purity}

NOTE: The following steps are necessary in order to check that the microbial concentration determined with the spectrophotometer in step 3.1.7 is correct and ensure that there was no microbial environmental contamination. A microbial environmental contamination can be easily detected by the appearance of various types of microbial colonies after $24 \mathrm{~h}$ of culture. Furthermore, no microbial contamination of the TSB medium during its manipulation in the decimal serial dilutions must be ensured with a negative TSB control plate.

1. Dispense the sterile TSB (prepared in step 3.1.4) into sterile microcentrifuge tubes using a micropipette with suitable autoclaved tips in aseptic conditions. One of them will be utilized as a negative TSB control.

2. Perform decimal serial dilutions with the microbial broth suspension utilized in step 3.1 .9 in the sterile microcentrifuge tubes containing TSB.

3. Spread $100 \mu \mathrm{L}$ of each dilution on TSA plates using a pre-sterilized Drigalski spatula or an alternative instrument. Also spread $100 \mu \mathrm{L}$ of TSB medium without microorganism (prepared in step 3.2.1) on a TSA plate as a negative TSB control plate.

4. Incubate aerobically the TSA plates aerobically at $37^{\circ} \mathrm{C}$ for $24 \mathrm{~h}$.

5. Count the number of colonies to check that the CFU/mL are similar to that determined in step 3.1.7.

6. Check that there is no microbial environmental contamination on the TSA plates with only one type of colonies.

7. Check that there is no microbial contamination on the TSB negative control plate showing no colonies

\section{Measurement of Antimicrobial Activity on Material Surfaces (Contact Method)}

NOTE: When surface contact might be the main antimicrobial mechanism of some advanced materials, the contact method can provide very useful information about the antimicrobial capacity of these materials. In this method, the microorganisms are placed directly onto the material surface and their growth inhibition can be determined after a certain amount of time.

\section{Initial procedure}

1. Prepare and autoclave TSB following the manufacturer's instructions.

2. Pour the TSB in a $50 \mathrm{~mL}$ pre-sterilized centrifuge tube with a pre-sterilized serological pipette under aseptic conditions using a Bunsen burner or a laminar flow hood.

3. Culture the different microorganisms to be tested aerobically overnight in the tube with TSB in an orbital shaker $(140 \mathrm{rpm})$ at $37^{\circ} \mathrm{C}$.

4. Dilute the overnight culture in $20 \mathrm{~mL}$ of TSB in a $50 \mathrm{~mL}$ pre-sterilized centrifuge tube to a concentration of approximately $10^{6} \mathrm{CFU} / \mathrm{mL}$ (determined with a spectrophotometer at $540 \mathrm{~nm}$ ).

NOTE: The culture and cuvette volumes to measure the absorbance must be selected according to the type of spectrophotometer utilized.

5. Perform decimal serial dilutions of this culture in sterile microcentrifuge tubes containing TSB. Spread $100 \mu \mathrm{L}$ of the culture on TSA plates and incubate it aerobically at $37^{\circ} \mathrm{C}$ for $24 \mathrm{~h}$.

6. Count the number of colonies to ensure an initial cell concentration of approximately $1 \times 10^{6} \mathrm{CFU} / \mathrm{mL}$

7. Place 4 control disks for microbial counting after $24 \mathrm{~h}$ and 4 sample disks of each type of the tested materials for microbial counting after $24 \mathrm{~h}$ in separates wells of a sterile 48-well plate.

8. Pipette $150 \mu \mathrm{L}$ of the microbial suspension onto each disk surface.

2. Microbial counting on the control and tested materials (after $\mathbf{2 4} \mathrm{h}$ )

1. After step 4.1 .6 , aerobically incubate the 4 sample disks remaining in the 48 -well plate at $37^{\circ} \mathrm{C}$ for $24 \mathrm{~h}$. NOTE: This $24 \mathrm{~h}$ incubation time can be modified in order to study the growth inhibition at shorter or longer times.

2. After $24 \mathrm{~h}$ of incubation, pipette $850 \mu \mathrm{L}$ of sterile PBS onto the surface of the 4 sample disks and mix it with the $150 \mu \mathrm{L}$ of the microbial suspension.

3. Collect the PBS/microbial suspension mixture and each disk from the 48-well plate and transfer them to a $10 \mathrm{~mL}$ pre-sterilized tube.

4. Vortex the PBS/microbial suspension mixture and each disk for $1 \mathrm{~min}$, sonicate it at $50 \mathrm{~Hz}$ for $5 \mathrm{~min}$ and vortex it again for $1 \mathrm{~min}$ to ensure that no viable microorganisms remain adhered to the material surface.

5. Perform decimal serial dilutions of each sonicated culture in sterile microcentrifuge tubes containing TSB and spread $100 \mu \mathrm{L}$ of the culture on TSA plates and incubate it aerobically at $37^{\circ} \mathrm{C}$ for $24 \mathrm{~h}$.

6. Count the number of colonies, which are the number of viable microorganisms on each sample and control disk surface. Express this number of viable cells in (CFU/mL).

7. Take a photograph of the final microbial culture for the sample and control disks. 


\section{Antimicrobial Results Analysis}

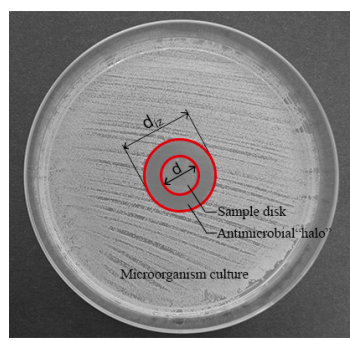

Figure 1: Measurements for the normalized width of the antimicrobial "halo". This panel shows the diameter of the inhibition zone $\left(d_{i z}\right)$ and the disk diameter $(d)$. Please click here to view a larger version of this figure.

1. Diffusion method results analysis

1. Measure the diameter of the inhibition zone $\left(d_{i z}\right)$ and the disk diameter $(d)$ (see Figure 1) with a digital caliper.

NOTE: The inhibition zone or antimicrobial "halo" is formed as a consequence of the microbial growth inhibition produced by the antimicrobial material disk (see Figure 1). It is possible to observe that there is a transparent ring zone close to the sample in comparison with the rest of the plate where the microorganisms have grown properly (opaque zone).

2. Determine the normalized width of the antimicrobial "halo" ( $\left.n w_{\text {halo }}\right)$ of each disk by applying equation (1).

(1) $n w_{\text {halo }}=\frac{\frac{d_{i z}-d}{2}}{d}$

3. Determine the mean and standard deviation of the normalized width of the antimicrobial "halo" with the 4 determined $n w_{\text {halo }}$ values of each sample.

4. Take a photograph of the final microbial culture with the material disk.

Note: The diameter of the inhibition zone $\left(d_{\mathrm{iz}}\right)$ and the disk diameter $(d)$ can also be measured from the photograph taken in step 5.1 .4 by using a suitable image processing software.

2. Contact method results analysis

1. Determine the number of viable microorganisms recovered according to equation (2).

(2)

$$
\underline{C \cdot D}
$$

$A$

NOTE: Here, $N$ is the number of viable microorganisms recovered per $\mathrm{cm}^{2}$ per test specimen; $C$ is the plate count; $D$ is the dilution factor; $A$ is the surface area of the test specimen in $\mathrm{cm}^{2}$ determined with the sample disk diameter.

2. Determine the loss of viability $(L V)$ to reflect the inhibition of cell growth by applying equation (3).

(3) $L V(\%)=\frac{C-S}{C} \cdot 100$

NOTE: Here, $\mathrm{C}$ is the average number of viable microorganisms $(N)$ in CFU/mL $\cdot \mathrm{cm}^{2}$, recovered from the control specimens after $24 \mathrm{~h}$; $\mathrm{S}$ is the number of viable microorganisms $(N)$ in $\mathrm{CFU} / \mathrm{mL} \cdot \mathrm{cm}^{2}$, recovered from the test specimens after $24 \mathrm{~h}$.

3. Determine the mean and standard deviation of the loss of viability with the 4 determined $L V(\%)$ values of each sample.

\section{Representative Results}

This protocol was employed, as an example, to test the antimicrobial capacity of 4 materials with different chemical natures against the 3 recommended microorganisms: Staphylococcus aureus, Escherichia coli, and Candida albicans. The results of the agar disk diffusion tests (diffusion method) exhibited non-antimicrobial activity for the first material (M1) as it occured in the control disk (C, image not shown) and increasing antibacterial activity against Gram-positive and Gram-negative bacteria for the other 3 materials M2, M3 and M4 (see Figure 2). 


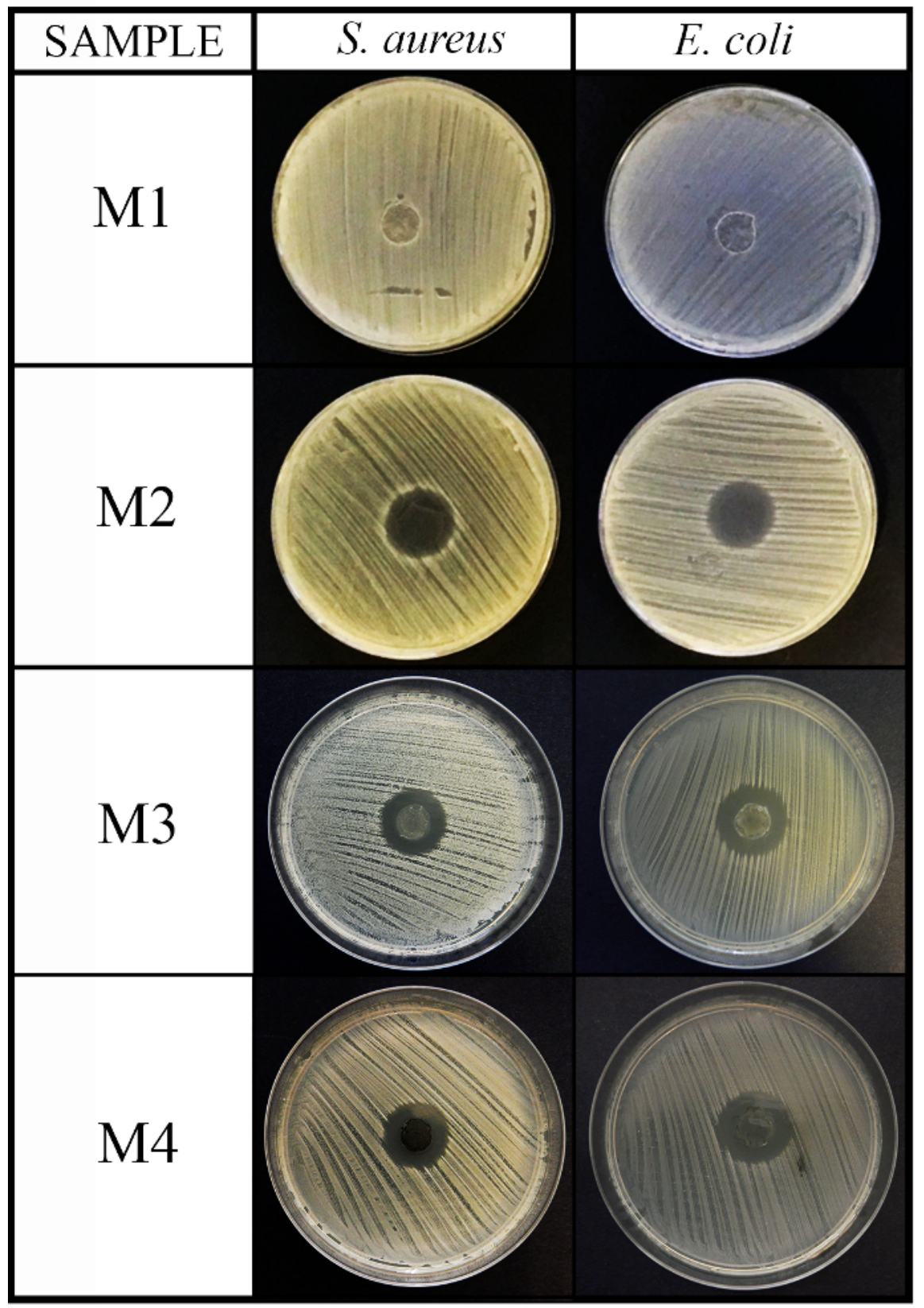

Figure 2: Antimicrobial diffusion method results. This panel shows the antimicrobial diffusion method for the 4 material (M1, M2, M3, and $\mathrm{M} 4$ ) disks (10 mm diameter $\times 1 \mathrm{~mm}$ thickness) against $S$. aureus and $E$. coli after $24 \mathrm{~h}$ of incubation. Please click here to view a larger version of this figure.

Figure 3 shows the different normalized widths of the antimicrobial "halo" ( $n w_{\text {halo }}$ ) for the different example materials M1, M2, M3, and M4 against Gram-positive and Gram-negative bacteria calculated with equation (1). However, none of the 4 materials were able to inhibit the growth of the yeast Candida albicans (images not shown). 


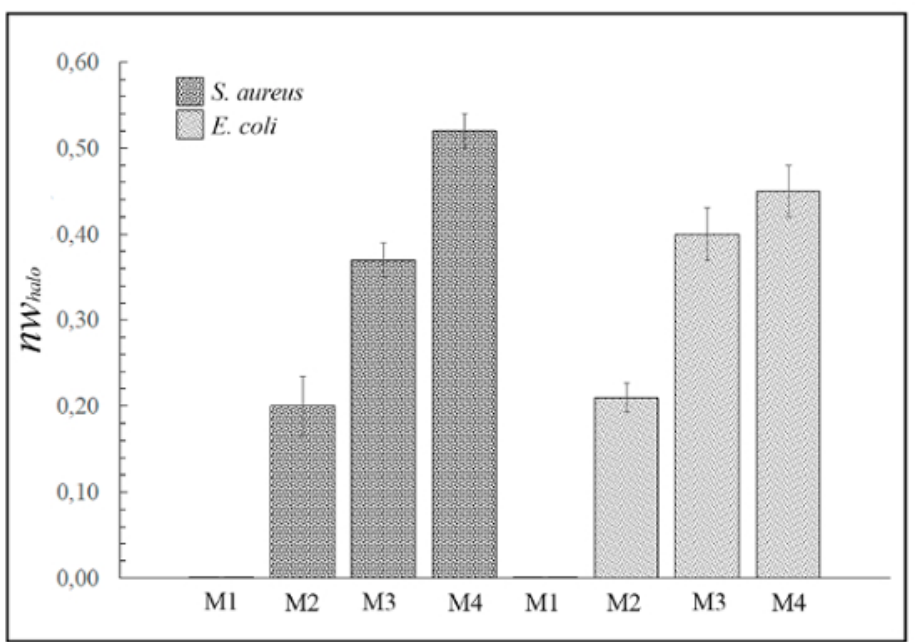

Figure 3: Antimicrobial diffusion "halo" results. This panel shows the normalized "halo" ( $\left.n w_{\text {halo }}\right)$ for each material (M1, M2, M3, and M4) disk (10 mm diameter $\times 1 \mathrm{~mm}$ thickness) against $S$. aureus and $E$. coli after $24 \mathrm{~h}$ of incubation. The differences are statistically significant $(p<0.01)$. However, sample M1 exhibited no antimicrobial activity. Please click here to view a larger version of this figure.

The results of the contact method also exhibited non-antimicrobial activity for the first material (M1) as it occurred in the control disk (C) and increasing antibacterial activity against Grampositive and Gram-negative bacteria for the other 3 materials (see Figure 4). 


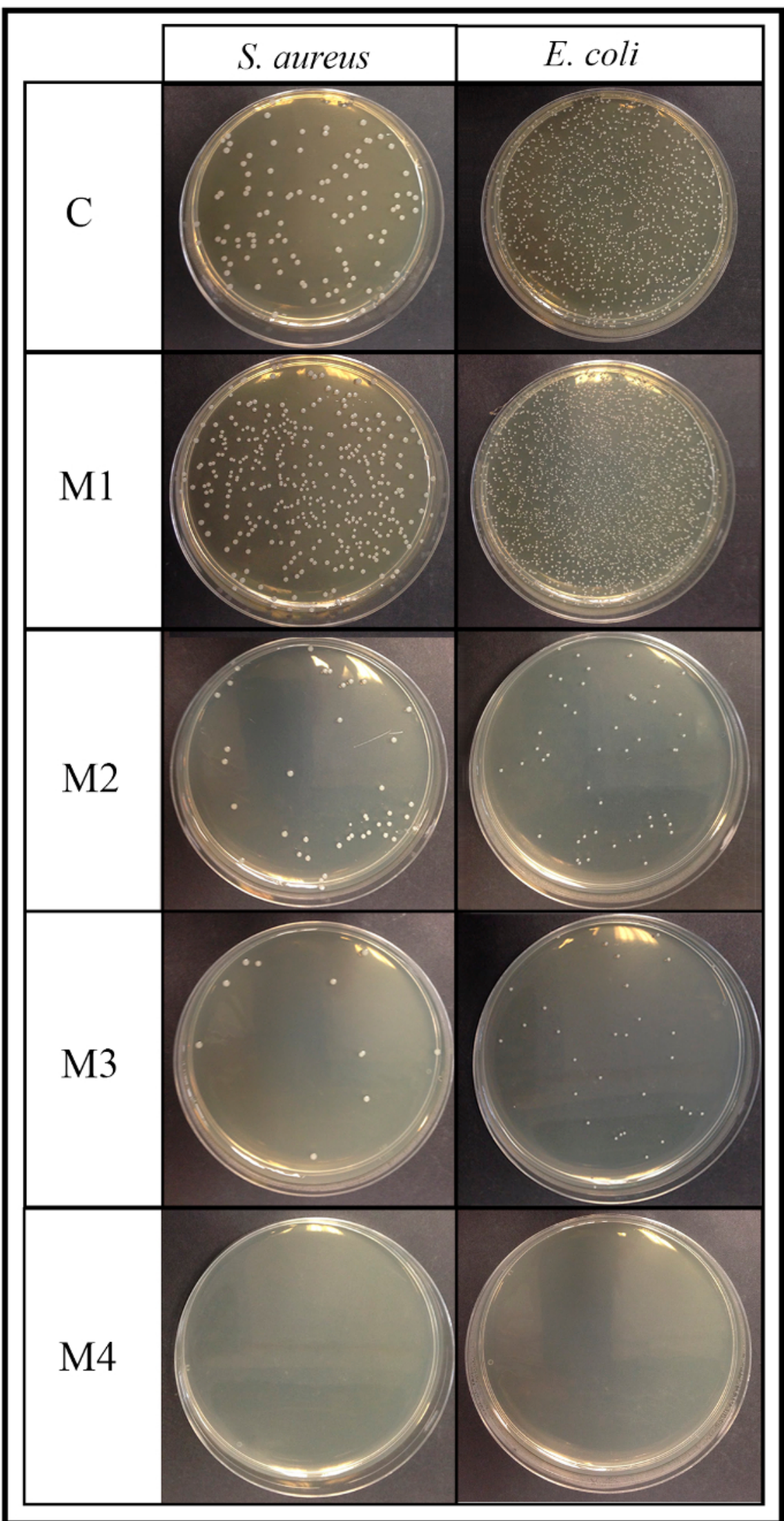


Figure 4: Antimicrobial contact method results. This panel shows the respective $90 \mathrm{~mm}$ plates of the $4 \mathrm{material}$ (M1, M2, M3, and M4) surface antimicrobial activity assay according to the ISO $22196: 2007$ after $24 \mathrm{~h}$ of incubation for S. aureus and E. coli (dilution factor of $10^{-4}$ ). C is the viable bacteria recovered from the control disk after $24 \mathrm{~h}$ of incubation. Please click here to view a larger version of this figure.

The loss of viability (\%) was determined by equation (2) and (3) as indicated in this protocol (see Figure 5).

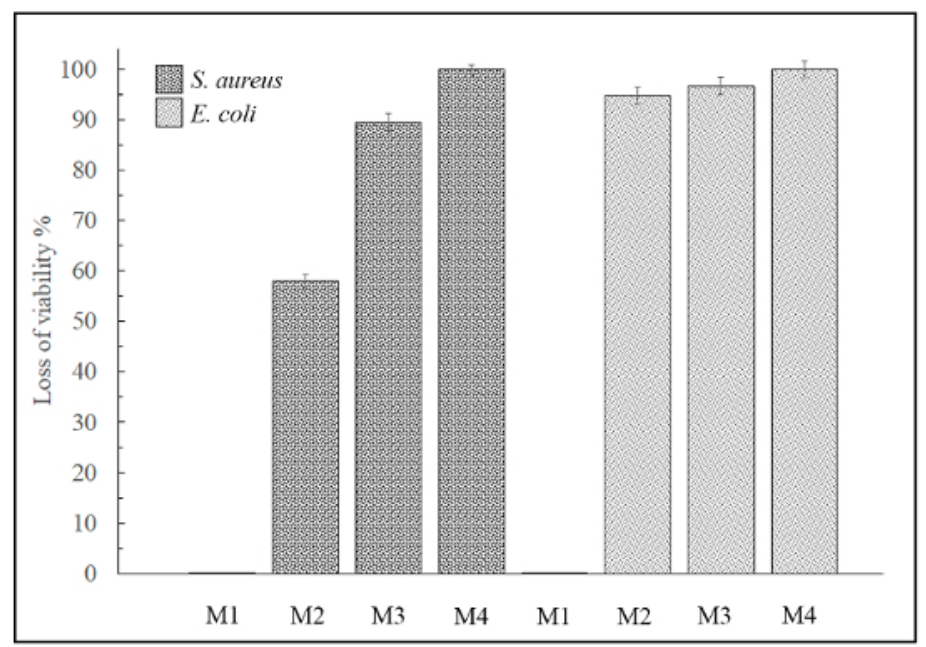

Figure 5: Loss of viability by contact method. This panel shows the loss of viability (\%) for M1, M2, M3, and M4 against S. aureus and E. coli on the material surfaces. Sample M1 exhibited no antimicrobial activity. Please click here to view a larger version of this figure.

However, none of the 4 materials were able to inhibit the growth of the yeast Candida albicans by the contact method either (images not shown). Therefore, 3 of these 4 advanced materials showed positive antimicrobial results against Gram-positive and Gram-negative bacteria and thus could be very useful for many bioengineering applications with high antibacterial activity requirements. However, none of the 4 materials were able to inhibit the yeast growth.

\section{Discussion}

The antimicrobial activity of new advanced materials can be analyzed by this easy-to-follow protocol consisting of 2 complementary procedures based on 2 existing methods: the agar disk diffusion test ${ }^{38}$ and the antimicrobial activity measured on material surfaces according to the ISO 22196:2007 norm $^{39}$

In this research field, many of the antimicrobial tests reported in the literature are highly assay-dependent. Therefore, it is very important to have detailed and consistent protocols in place across laboratories. This article is a step in that direction. Furthermore, it could be very helpful for many researchers who are less experienced in this field and require in-depth, step-by-step procedures to follow for accurate results.

This protocol can be used with many types of materials cut into disk shapes of 10-mm diameter. Brittle materials can be swollen in a suitable solvent for $1 \mathrm{~h}$ to render the cutting process easier. Thus, hydrophilic materials such as alginates can be hydrated in autoclaved distilled water. Other solvents, such as ethanol, ketone, and dichloromethane, can be employed to swell hydrophobic materials for $1 \mathrm{~h}$ before cutting them. However, some materials such as poly(3-hydroxybutyrate-co-3-hydroxyvalerate) do not need to be swollen and they can be cut directly. After that, it is very important to dry the sample material disks in a vacuum oven and sterilize each specimen with ethanol and UV radiation for $1 \mathrm{~h}$ to avoid any contamination risk.

This protocol recommends TSA and TSB as culture media and the use of pure cultures of 3 microorganisms to reach a broad range of microorganisms: the Gram-positive bacteria Staphylococcus aureus, the Gram-negative bacteria Escherichia coli, and the yeast Candida albicans. However, alternative culture media and other microorganisms in need of different incubation conditions could also be used with this protocol. Sometimes, only 1 microorganism is tested to have an initial idea of the antimicrobial activity of a new material.

The materials showing strong antimicrobial activity against the recommended 3 different types of microorganisms should also be tested against antibiotic-resistant pathogens such as methicillin-resistant Staphylococcus epidermidis (MRSE), which have been successfully utilized with this protocol. Other important drug-resistant microorganisms which are causing much concern are the Gram-positive methicillin-resistant Staphylococcus aureus (MRSA) and vancomycin-resistant Enterococci (VRE), and the Gram-negative Pseudomonas aeruginosa ${ }^{40,41}$.

Biofilm inhibition and the antimicrobial activity of materials against other types of microorganisms such as viruses and parasites cannot be tested with this protocol. However, this protocol provides a very useful starting point for an antimicrobial study of a new advanced material.

In the antimicrobial agar disk diffusion test, a critical step occurs when the sample disk has to be placed in the center of the plate because some materials fold as soon as they get in contact with the agar media. In this case, it is recommended to use a sterile pair of tweezers to carefully unfold the sample. On the other hand, in the contact method, it is critical to wash the control and sample disks very well with PBS by pipetting them four times followed by a vigorous vortexing and sonication in order to ensure that no viable microorganisms remain adhered to the material surface. 
This video protocol can be utilized in many bioengineering applications, such as bioprocess engineering, tissue engineering, controlled drug delivery, packaging materials, wastewater treatment, and agriculture, which use biomaterials with a highly desirable antimicrobial capacity.

The results obtained with this protocol are qualitative (the images) and quantitative (the normalized width of the antibacterial "halo" and the loss of viability) with a good analysis of its reproducibility (mean \pm standard deviation). When comparing different materials, these mean values obtained with the diffusion and contact method results analysis must be analyzed by one-way ANOVA, followed by Turkey's post hoc analysis, in order to study if they are, statistically, significantly different $(p<0.01)$.

\section{Disclosures}

The authors have nothing to disclose.

\section{Acknowledgements}

The authors would like to acknowledge the Universidad Católica de Valencia San Vicente Mártir for the financial support for this work through the 2017-231-001UCV and 2018-231-001UCV grants.

\section{References}

1. Sydnor, E.R.M., Perl, T.M. Hospital epidemiology and infection control in acute-care settings. Clin Microbiol Rev. 24 (1), 141-173 (2011).

2. Chessa, D. et al.Staphylococcus aureus and Staphylococcus epidermidis Virulence Strains as Causative Agents of Persistent Infections in Breast Implants. PLoS One. 11 (1), e0146668 (2016).

3. Pandey, H., Parashar, V., Parashar, R., Prakash, R., Ramteke, P.W., Pandey, A.C. Controlled drug release characteristics and enhanced antibacterial effect of graphene nanosheets containing gentamicin sulfate. Nanoscale. 3 (10), 4104 (2011).

4. Jia, Z., Shen, D., Xu, W. Synthesis and antibacterial activities of quaternary ammonium salt of chitosan. Carbohydr Res. 333 (1), 1-6 (2001).

5. Liu, Y., Wang, X., Yang, F., Yang, X. Excellent antimicrobial properties of mesoporous anatase $\mathrm{TiO}_{2}$ and $\mathrm{Ag} / \mathrm{TiO}{ }_{2} \mathrm{composite}$ films. Microporous Mesoporous Mater. 114 (1-3), 431-439 (2008).

6. Wang, L., Chen, J., Shi, L., Shi, Z., Ren, L., Wang, Y. The promotion of antimicrobial activity on silicon substrates using a "click" immobilized short peptide. Chem Commun (Camb). 50 (8), 975-977 (2014).

7. Kümmerer, K. Resistance in the environment. J Antimicrob Chemother. 54 (2), 311-320 (2004).

8. Ng, V.W.L. et al. Antimicrobial hydrogels: A new weapon in the arsenal against multidrug-resistant infections. Adv Drug Deliv Rev. 78, 46-62 (2014).

9. Hegstad, K., Langsrud, S., Lunestad, B.T., Scheie, A.A., Sunde, M., Yazdankhah, S.P. Does the Wide Use of Quaternary Ammonium Compounds Enhance the Selection and Spread of Antimicrobial Resistance and Thus Threaten Our Health? Microb Drug Resist. 16 (2), 91-104 (2010).

10. Rana, D., Matsuura, T. Surface modifications for antifouling membranes. Chem Rev. 110 (4), 2448-2471 (2010).

11. Lok, C.N. et al. Proteomic analysis of the mode of antibacterial action of silver nanoparticles. J Proteome Res. 5 (4), $916-924$ (2006).

12. Chen, X., Schluesener, H.J. Nanosilver: A nanoproduct in medical application. Toxicol Lett. 176 (1), 1-12 (2008).

13. Ahamed, M., AlSalhi, M.S., Siddiqui, M.K.J. Silver nanoparticle applications and human health. Clin Chim Acta. 411 (23-24), 1841-1848 (2010).

14. Yeaman, M.R. Mechanisms of Antimicrobial Peptide Action and Resistance. Pharmacol Rev. 55 (1), $27-55$ (2003).

15. McLean, D.T.F., Lundy, F.T., Timson, D.J. IQ-motif peptides as novel anti-microbial agents. Biochimie. 95 (4), $875-880$ (2013).

16. Brogden, K.A. Antimicrobial peptides: Pore formers or metabolic inhibitors in bacteria? Nat Rev Microbiol. 3 (3), 238-250 (2005)

17. Ghosh, C. et al. Small molecular antibacterial peptoid mimics: The simpler the better! J Med Chem. 57 (4), 1428-1436 (2014).

18. Chongsiriwatana, N.P. et al. Peptoids that mimic the structure, function, and mechanism of helical antimicrobial peptides. Proc Natl Acad Sci. 105 (8), 2794-2799 (2008).

19. Chen, Y., Mant, C.T., Farmer, S.W., Hancock, R.E.W., Vasil, M.L., Hodges, R.S. Rational design of alpha-helical antimicrobial peptides with enhanced activities and specificity/therapeutic index. J Biol Chem. 280 (13), 12316-12329 (2005).

20. Porter, E.A., Wang, X., Lee, H.S., Weisblum, B., Gellman, S.H. Non-haemolytic beta-aminoacid oligomers. Nature. 404 (6778), 565 (2000).

21. Gao, Q., Li, P., Zhao, H., Chen, Y., Jiang, L., Ma, P.X. Methacrylate-ended Polypeptides and Polypeptoids for Antimicrobial and Antifouling Coatings. Polym Chem. 8 (41), 6386-6397 (2017).

22. Serrano-Aroca, Á., Gómez-Ribelles, J.L., Monleón-Pradas, M., Vidaurre-Garayo, A., Suay-Antón, J. Characterisation of macroporous poly(methyl methacrylate) coated with plasma-polymerised poly(2-hydroxyethyl acrylate). Eur Polym J. 43 (10), $4552-4564$ (2007).

23. Serrano-Aroca, Á., Monleón-Pradas, M., Gómez-Ribelles, J.L. Plasma-induced polymerisation of hydrophilic coatings onto macroporous hydrophobic scaffolds. Polymer (Guildf). 48 (7), 2071-2078 (2007).

24. Serrano-Aroca, Á., Monleón-Pradas, M., Gómez-Ribelles, J.L., Rault, J. Thermal analysis of water in reinforced plasma-polymerised poly(2hydroxyethyl acrylate) hydrogels. Eur Polym J. 72, 523-534 (2015).

25. Monleón-Pradas, M., Gómez-Ribelles, J.L., Serrano-Aroca, Á., Gallego-Ferrer, G., SuayAntón, J., Pissis, P. Interaction between water and polymer chains in poly(hydroxyethyl acrylate) hydrogels. Colloid Polym Sci. 279 (4), 323-330 (2001).

26. Serrano-Aroca, Á., Monleón-Pradas, M., Gómez-Ribelles, J.L. Effect of crosslinking on porous poly(methyl methacrylate) produced by phase separation. Colloid Polym Sci. 286 (2), 209-216 (2008).

27. Monleón-Pradas, M., Gómez-Ribelles, J.L., Serrano-Aroca, Á., Gallego Ferrer, G., Suay Antón, J., Pissis, P. Porous poly (2-hydroxyethyl acrylate) hydrogels. Polymer (Guildf). 42 (10), 4667-4674 (2001).

28. Serrano-Aroca, Á., Monleón-Pradas, M., Gómez-Ribelles, J.L. Macroporous poly(methyl methacrylate) produced by phase separation during polymerisation in solution. Colloid Polym Sci. 285 (7), 753-760 (2007).

29. Serrano-Aroca, Á., Llorens-Gámez, M. Dynamic mechanical analysis and water vapour sorption of highly porous poly(methyl methacrylate). Polymer (Guildf). 125, 58-65 (2017). 
30. Serrano-Aroca, Á., Campillo-Fernández, A.J., Gómez-Ribelles, J.L., Monleón-Pradas, M., Gallego-Ferrer, G., Pissis, P. Porous poly(2hydroxyethyl acrylate) hydrogels prepared by radical polymerisation with methanol as diluent. Polymer (Guildf). 45 (26), $8949-8955$ (2004).

31. Rodríguez-Hernández, J.C., Serrano-Aroca, Á., Gómez-Ribelles, J.L., Monleón-Pradas, M. Three-dimensional nanocomposite scaffolds with ordered cylindrical orthogonal pores. J Biomed Mater Res - Part B: Appl Biomater. 84 (2), 541-549 (2008).

32. Brígido-Diego, R. et al. Acrylic scaffolds with interconnected spherical pores and controlled hydrophilicity for tissue engineering. $J$ Mater Sci Mater Med. 40 (18), $4881-4887$ (2005).

33. Serrano-Aroca, Á., Ruiz-Pividal, J.F., Llorens-Gámez, M. Enhancement of water diffusion and compression performance of crosslinked alginate with a minuscule amount of graphene oxide. Sci Rep. 7, 11684 (2017).

34. Serrano-Aroca, Á., Deb, S. Synthesis of irregular graphene oxide tubes using green chemistry and their potential use as reinforcement materials for biomedical applications. PLoS One. 12 (9), e0185235 (2017).

35. Sánchez-Correa, F., Vidaurre-Agut, C., Serrano-Aroca, A., Campillo-Fernández, A.J. Poly(2-hydroxyethyl acrylate) hydrogels reinforced with graphene oxide: remarkable improvement of water diffusion and mechanical properties. J Appl Polym Sci. 46158 (2018).

36. Serrano-Aroca, Á., Iskandar, L., Deb, S. Green synthetic routes to alginate-graphene oxide composite hydrogels with enhanced physical properties for bioengineering applications. Eur Polym J. 103, 198-206 (2018).

37. Llorens-Gámez, M., Serrano-Aroca, Á. Low-Cost Advanced Hydrogels of Calcium Alginate/Carbon Nanofibers with Enhanced Water Diffusion and Compression Properties. Polymers (Basel). 10 (4), 405, (2018).

38. Bauer, A.W., Kirby, W.M.M., Sherris, J.C., Turck, A.M. Antibiotic susceptibility testing by a standardized single disk method. Am J Clin Pathol. 45, 493-496 (1966).

39. ISO Specification 22196: measurement of antibacterial activity on plastics surfaces. (2007).

40. Taubes, G. The bacteria fight back. Science. (80-). 321 (5887), 356-361, (2008).

41. Boucher, H.W. et al. 10 × '20 progress--development of new drugs active against Gram-negative bacilli: an update from the infectious diseases society of America. Clinical Infectious Diseases. 56 (12), 1685-1694 (2013). 